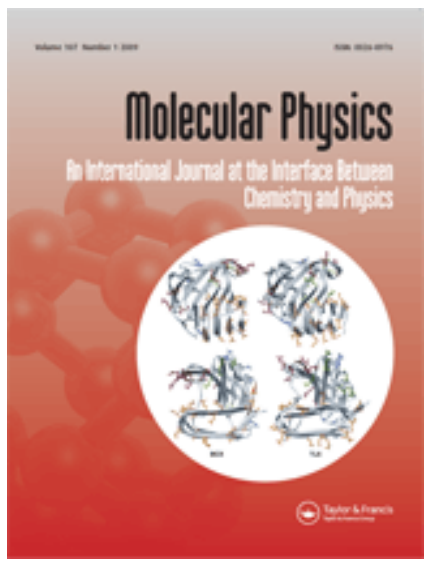

\title{
14N Pulsed nuclear quadrupole resonance. 4. Two-pulse sequences for the determination of $\mathrm{T} 1$ and $\mathrm{T} 2$ relaxation times.
}

\begin{tabular}{|r|l|}
\hline Journal: & Molecular Physics \\
\hline Manuscript ID: & TMPH-2009-0052.R3 \\
\hline Manuscript Type: & Full Paper \\
\hline Author: & $10-$ Sep-2009 \\
\hline Complete List of Authors: & $\begin{array}{l}\text { CANET, Daniel; Nancy-Université, Méthodologie RMN } \\
\text { Ferrari, Maude; Nancy-Université, Méthodologie RMN, Faculté des } \\
\text { sciences }\end{array}$ \\
\hline Keywords: & $\begin{array}{l}\text { Nuclear Quadrupole Resonance, Nitrogen-14, spin relaxation times, } \\
\text { two-pulse sequences }\end{array}$ \\
\hline \multicolumn{2}{|c|}{} \\
\hline $\begin{array}{l}\text { Note: The following files were submitted by the author for peer review, but cannot be converted } \\
\text { to PDF. You must view these files (e.g. movies) online. }\end{array}$ \\
\hline New WinZip File.zip
\end{tabular}

\section{今 scholarONE" \\ Manuscript Central}


Abstract

A general theory, based on density matrix calculations, has been developed for the special case of a two-pulse sequence applied to spin $1\left({ }^{14} \mathrm{~N}\right)$ Nuclear Quadrupole Resonance (NQR) of a powder sample. It is shown that the homolog of the NMR inversion-recovery experiment leads easily to the spin-lattice relaxation time $T_{1}$ (associated with the diagonal elements of the density matrix) provided that an appropriate phase cycling is used. Conversely, in spite of two-step phase cycling schemes adapted to spin-spin relaxation measurements, the homolog of the NMR Hahn spin-echo sequence may pose some problems if the results are displayed in the magnitude mode. First, at short decay times, the echo may be corrupted by unwanted signals. Secondly, in that case, the amplitude of the resulting signal can evolve unexpectedly and differently as a function of the phase of the second pulse. Thirdly, at long decay times, the echo maximum occurs earlier than expected. All these problems in principle disappear with a complete four-step phase cycling scheme and the echo decay curve yields reliably the spinspin relaxation time $T_{2}$ (associated with off-diagonal elements). This theory allowed us to exploit many test experiments performed at different frequencies on hexamethylenetetramine (HMT) and sodium nitrite.

Keywords: Nuclear Quadrupole Resonance; Nitrogen-14; spin relaxation times; two-pulse sequences 


\section{Introduction}

In NMR, the measurement of spin relaxation times rests on well-known and well-understood methods, perfectly reliable at the present time [1]. The so-called spin-lattice relaxation time $T_{1}$ (or longitudinal relaxation time in NMR), is usually deduced from the inversion-recovery experiment: a $180^{\circ}$ inverting radio-frequency (rf) pulse, followed by an evolution period $(\tau)$ and terminated by a $90^{\circ}$ read-pulse which produces the NMR signal proportional to $1-k \exp \left(-\tau / T_{1}\right), k$ being an experimental parameter $(1 \leq k \leq 2)$. The simplest experiment for measuring the spin-spin relaxation time $T_{2}$ (or transverse relaxation time in NMR) is the Hahn spin echo : a $90^{\circ}$ pulse followed by a evolution period $(\tau)$, then a $180^{\circ}$ pulse which entails the formation of a spin echo at a time $\tau$ later. The amplitude of this echo decreases according to $\exp \left(-2 \tau / T_{2}\right)$.

More or less complicated sequences have been used to measure the ${ }^{14} \mathrm{~N}$ NQR spin-lattice relaxation time $T_{1}$ [2-5] but inversion-recovery type sequences have been mentioned scarcely [3]. Conversely, the ${ }^{14} \mathrm{~N}$ NQR spin-spin relaxation has been often investigated with sequences of the Hahn spin-echo type [5-8]. It turns out that the transposition of NMR sequences to NQR of powder samples is not straightforward for several reasons. First, there is evidently no Zeeman term in pure NQR, thus no preferential direction. This prevents us to resort to the concept of the rotating frame which is invaluable in NMR for predicting the effects of radio frequency pulses. Thus, one is forced to rely on a density matrix treatment. If the density matrix is built on the Hamiltonian eigenstate basis, $T_{1}$ is related to diagonal elements and therefore to the recovery toward equilibrium, whereas off-diagonal elements decay according to $T_{2}$. The second difficulty arises still from the lack of a preferential direction and from the random orientation of the microcrystallites constituting the powder sample. The first consequence of this feature is that the rf pulses which correspond respectively to the first maximum and to the first zero of nutation curve (signal amplitude as a function of the pulse length) are not true $90^{\circ}$ and $180^{\circ}$ pulses [9]. They will be denoted in the following pseudo $90^{\circ}$ and pseudo $180^{\circ}$ pulses, respectively. Although several theoretical approaches have been devoted to the two-pulse sequences in NQR [10-11], none of them takes really into account that the equivalent of the flip angle is different from one microcrystallite to the other. We have recognized this property in previous papers which were devoted either to nutation curves [12-13] or to the optimal conditions of data averaging [14]. In particular, we showed [13] that 


\section{Theory}

Let us consider a sequence made of two rf pulses (separated by a time interval $\tau$ ) of durations $\delta_{0}$ and $\delta_{1}$, respectively (figure1). Signal acquisition is supposed to start at $t=0$, immediately after the second pulse. Let us denote by $\alpha_{0}$ and $\alpha_{1}$ the standard (NMR) flip angle corresponding to these two pulses:

$$
\alpha_{0,1}=\gamma B_{1} \delta_{0,1}
$$

$\gamma$ being the gyromagnetic ratio and $B_{1}$ the amplitude of the ratio frequency field. At the level of each microcrystallite, the effective flip angle is given by: 


$$
\beta_{0,1}=\gamma B_{1} \delta_{0,1} \times f(\theta, \varphi)
$$

$\theta$ and $\varphi$ are the usual polar angles and $f(\theta, \varphi)$ indicates the direction of the $B_{1}$ axis with respect to the PAS (Principal Axis System) of the electric field gradient tensor. As shown in previous papers [12-13], $f(\theta, \varphi)$ is expressed as follows, according to the type of transitions:

- Single line in the case of an axially symmetry tensor $f_{\text {axial }}(\theta, \varphi)=\sin \theta$ (due to the fact that, if $z$ is the symmetry axis, the single transition is of the type $\omega_{x}[13]$ )

- Line $\omega_{x}$ in the general case $f_{x}(\theta, \varphi)=\sin \theta \cos \varphi$

- Line $\omega_{y}$ in the general case $f_{y}(\theta, \varphi)=\sin \theta \sin \varphi$

- Line $\omega_{z}$ in the general case $f_{z}(\theta, \varphi)=\cos \theta$

In the general case the three lines are in the order $\omega_{x}>\omega_{y}>\omega_{z}$. Also, we have shown previously that, for any line, the evolution of the system can be accounted by a $2 \times 2$ density matrix. At equilibrium, a working form of the density matrix can be written as

$$
\sigma_{0}=\left(\begin{array}{cc}
0 & 0 \\
0 & m_{0}
\end{array}\right)
$$

In fact, the equilibrium density matrix has the form

$$
\sigma_{e q}=\left(\begin{array}{cc}
p_{0}-\frac{m_{0}}{2} & 0 \\
0 & p_{0}+\frac{m_{0}}{2}
\end{array}\right)
$$

Where $p_{0}$ is the population of each level in the absence of quadrupolar interaction and $m_{0}$ is the population difference, proportional to the line intensity. $\sigma_{e q}$ can therefore be expanded according to

$$
\sigma_{e q}=\left(p_{0}-\frac{m_{0}}{2}\right)\left(\begin{array}{ll}
1 & 0 \\
0 & 1
\end{array}\right)+\left(\begin{array}{cc}
0 & 0 \\
0 & m_{0}
\end{array}\right)=\left(p_{0}-\frac{m_{0}}{2}\right) E+\sigma_{0}
$$

$E$ being the identity matrix. Since the first term is invariant under any transformation, one has just to deal with $\sigma_{0}$ in order to follow the evolution of the spin system. 
This is quite comparable to the density matrix of a spin $1 / 2$ system. The effect of a radiofrequency pulse is also similar to a spin $1 / 2$. As an example, $\sigma^{+}$and $\sigma^{-}$denoting the density matrix just after and just before the rf pulse, one has

$$
\sigma_{+}=R_{0,90}^{\dagger}(\beta) \sigma_{-} R_{0,90}(\beta)
$$

( + : transpose complex conjugate). $R_{0,90}$ is a so-called propagator (or rotation matrix) with its subscript associated with the phase of the ratio frequency field $\left(0^{\circ}\right.$ or $\left.90^{\circ}\right) . \beta$ is the effective flip angle (see (2)) which is different from one microcrystallite to the other. A phase shift of $180^{\circ}$ amounts to change $\beta$ into $-\beta$. One has for the two basic rotation matrices:

$$
R_{0}(\beta)=\left(\begin{array}{cc}
\cos \frac{\beta}{2} & -i \sin \frac{\beta}{2} \\
-i \sin \frac{\beta}{2} & \cos \frac{\beta}{2}
\end{array}\right) ; R_{90}(\beta)=\left(\begin{array}{cc}
\cos \frac{\beta}{2} & +\sin \frac{\beta}{2} \\
-\sin \frac{\beta}{2} & \cos \frac{\beta}{2}
\end{array}\right)
$$

We shall assume zero phase for the first pulse and we shall consider the two possibilities ( $0^{\circ}$ and $90^{\circ}$ phases) for the second pulse. Assuming equilibrium at the onset, and denoting by $\sigma_{0}^{+}$the density matrix just after this pulse, one has

$$
\sigma_{0}^{+}=m_{0}\left(\begin{array}{cc}
\sin ^{2} \frac{\beta_{0}}{2} & \left(\frac{i}{2}\right) \sin \beta_{0} \\
-\left(\frac{i}{2}\right) \sin \beta_{0} & \cos ^{2} \frac{\beta_{0}}{2}
\end{array}\right)
$$

We shall now introduce relaxation phenomena during the evolution time $\tau$. Spin-spin relaxation is only involved in the off-diagonal elements (coherences). As far as the genuine relaxation $\left(T_{2}\right)$ is concerned, this amounts to multiply the considered off-diagonal element by $e^{\frac{-\tau}{T_{2}}}$. Now, to account for the line-form factor $\left(T_{2}^{*}\right)$ (due, for instance, to temperature variations affecting the quadrupolar interaction), we need to multiply the $(1,2)$ element by $e^{i \omega^{*} \tau}$ and the $(2,1)$ element by $e^{-i \omega^{*} \tau}$ with $\frac{\omega^{*}}{2 \pi}$ somewhere in the interval $\left[0,\left(\frac{1}{T_{2}^{*}}-\frac{1}{T_{2}}\right)\right]$, 
$\frac{1}{\pi T_{2}^{*}}$ being the line width at half-height in the case of a Lorentzian line. This can be an approximation in practical situations since NQR lines look rather Gaussian. $\omega^{*}$ can be considered as the frequency difference (in rad. $s^{-1}$ ) of an extra resonance with respect to the main resonance $\omega_{0}$, the oscillating factors related to the main resonance being omitted in the above density matrices. $\omega_{0} / 2 \pi$ is of the order of some MHz whereas $\omega^{*} / 2 \pi$ is of the order of some tens or hundreds of Hz. Of course, we shall consider at the end a distribution of $\omega^{*}$. Spin-lattice relaxation concerns evidently diagonal elements (populations). This type of relaxation, defined by a time constant denoted $T_{1}$, tends to restore populations toward their equilibrium values. Thus, the $(1,1)$ element is simply multiplied by $e^{\frac{-\tau}{T_{1}}}$ whereas, the $(2,2)$ element must be written as $m_{0}+\left(m(0)-m_{0}\right) e^{\frac{-\tau}{T_{1}}}, m(0)$ being the value of this element at the beginning of the evolution period (for instance, from (5), $m(0)=m_{0} \cos ^{2} \frac{\beta}{2}$ ).

The density matrix prior to the second pulse $\left(\sigma_{1}^{-}\right)$can be written as:

$$
\sigma_{1}^{-}=m_{0}\left(\begin{array}{cc}
\sin ^{2} \frac{\beta_{0}}{2} e^{\frac{-\tau}{T_{1}}} & \left(\frac{i}{2}\right) \sin \beta_{0} e^{\frac{-\tau}{T_{2}}} e^{i \omega^{*} \tau} \\
-\left(\frac{i}{2}\right) \sin \beta_{0} e^{\frac{-\tau}{T_{2}}} e^{-i \omega^{*} \tau} & 1-\sin ^{2} \frac{\beta_{0}}{2} e^{\frac{-\tau}{T_{1}}}
\end{array}\right)
$$

Upon application of a radio-frequency pulse $\beta_{1}$ of $0^{\circ}$ phase, we obtain for the density matrix at the end of the pulse 


$$
\sigma_{1}^{+}\left[\left(\beta_{1}\right)_{0}\right]=m_{0}\left(\begin{array}{l:l}
\sin ^{2} \frac{\beta_{0}}{2} \cos ^{2} \frac{\beta_{1}}{2} e^{\frac{-\tau}{T_{1}}} & \frac{i}{2} \sin \beta_{0} \cos ^{2} \frac{\beta_{1}}{2} e^{\frac{-\tau}{T_{2}}} e^{i \omega^{*} \tau} \\
+\left(1-\sin ^{2} \frac{\beta_{0}}{2} e^{\frac{-\tau}{T_{1}}}\right) \sin ^{2} \frac{\beta_{1}}{2} & -\frac{i}{2} \sin \beta_{0} \sin ^{2} \frac{\beta_{1}}{2} e^{\frac{-\tau}{T_{2}}} e^{-i \omega^{*} \tau} \\
+\frac{1}{2} \sin \beta_{0} \sin \beta_{1} \cos \omega^{*} \tau e^{\frac{-\tau}{T_{2}}} & +i \sin \beta_{1}\left(\frac{1}{2}-\sin ^{2} \frac{\beta_{0}}{2} e^{\frac{-\tau}{T_{1}}}\right) \\
\hdashline \frac{i}{2} \sin \beta_{0} \sin ^{2} \frac{\beta_{1}}{2} e^{\frac{-\tau}{T_{2}}} e^{i \omega^{*} \tau} & \sin ^{2} \frac{\beta_{0}}{2} \sin ^{2} \frac{\beta_{1}}{2} e^{\frac{--\bar{\tau}}{T_{1}}} \\
-\frac{i}{2} \sin \beta_{0} \cos ^{2} \frac{\beta_{1}}{2} e^{\frac{-\tau}{T_{2}}} e^{-i \omega^{*} \tau} & +\left(1-\sin ^{2} \frac{\beta_{0}}{2} e^{\frac{-\tau}{T_{1}}}\right) \cos ^{2} \frac{\beta_{1}}{2} \\
-i \sin \beta_{1}\left(\frac{1}{2}-\sin ^{2} \frac{\beta_{0}}{2} e^{\frac{-\tau}{T_{1}}}\right) & -\frac{1}{2} \sin \beta_{0} \sin \beta_{1} \cos \omega^{*} \tau e^{\frac{-\tau}{T_{2}}}
\end{array}\right)
$$

and for a phase of $90^{\circ}$

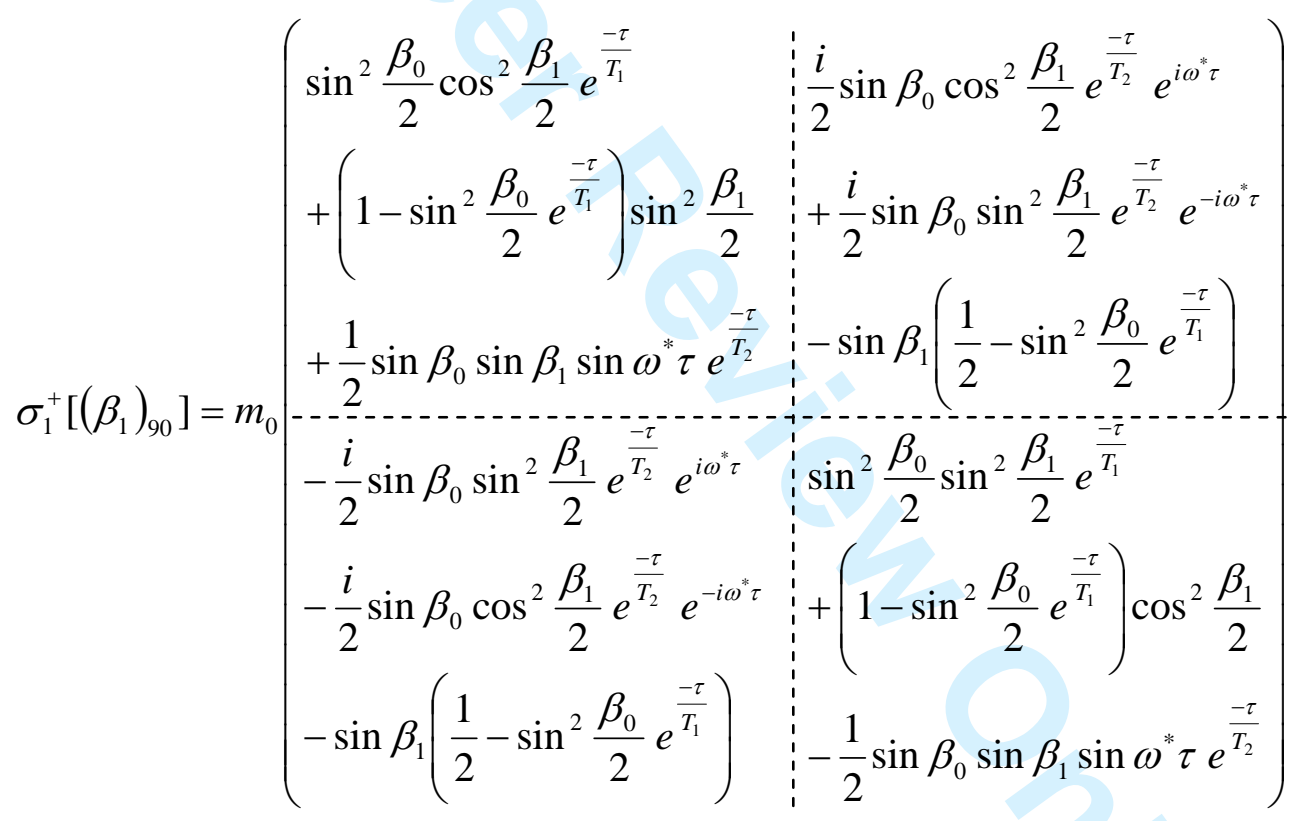

\section{1. $T_{1}$ measurements}

In both matrices, the measured signal is represented by the off-diagonal elements (one being the complex conjugate of the other). As already indicated, they should be ultimately multiplied by $e^{i \omega_{0} t}$ for the $(1,2)$ element and $e^{-i \omega_{0} t}$ for the element $(2,1)$, $\omega_{0}$ being the resonance frequency (in rad.s ${ }^{-1}$ ). In these elements, there is only one term which depends on 
$T_{1}: \sin \beta_{1}\left(\frac{1}{2}-\sin ^{2} \frac{\beta_{0}}{2} e^{\frac{-\tau}{T_{1}}}\right)$. Therefore, if we want to measure $T_{1}$ properly, we have to cancel the other two terms. This can be done very easily by a very simple phase cycling (phases are indicated in degrees):

\begin{tabular}{lll}
$\beta_{0}$ & $\beta_{1}$ & Acq \\
\hline $0^{\circ}$ & $0^{\circ}$ & + \\
$180^{\circ}$ & $0^{\circ}$ & +
\end{tabular}

Or alternatively

\begin{tabular}{ccc}
$\beta_{0}$ & $\beta_{1}$ & Acq \\
\hline $0^{\circ}$ & $0^{\circ}$ & + \\
$0^{\circ}$ & $180^{\circ}$ & -
\end{tabular}

The above procedure rests on similar principles as the ones proposed by Rudakov and Mikhaltsvitch [3]. Moreover, we can notice that the maximum would be obtained for $\beta_{0}=\pi$ and $\beta_{1}=\frac{\pi}{2}$ (which is the classical inversion recovery experimentation). Very naturally, we choose pulse lengths corresponding respectively to the first zero in the nutation curve for $\delta_{0}$ (equivalent in NMR to a $\pi$ flip angle; pseudo $180^{\circ}$ pulse) and the first maximum for $\delta_{1}$ (equivalent in NMR to a $\pi / 2$ flip angle; pseudo $90^{\circ}$ pulse). Of course, we would have to calculate $\beta_{0}$ and $\beta_{1}$ for each microcrystallite and perform an average over the whole sample. However, as we are primarily interested in the $T_{1}$ value, it suffices to extract the time constant from the signal evolution as a function of $\tau$.

\section{2. $T_{2}$ measurements}

\section{General Considerations}


It can be seen that $T_{2}$ shows up only in the first two term of the off-diagonal elements (coherences) of (7) and (8). If we want to measure $T_{2}$, we have at least to remove the third term of these elements (which depends on $T_{1}$ ). This can be easily accomplished by the following phase cycle:

$$
\begin{array}{ccc}
\beta_{0} & \beta_{1} & \text { Acq } \\
\hline 0^{\circ} & 0^{\circ} & + \\
0^{\circ} & 180^{\circ} & +
\end{array}
$$

Or alternatively

$$
\begin{array}{lll}
\beta_{0} & \beta_{1} & \text { Acq } \\
\hline 0^{\circ} & 0^{\circ} & + \\
180^{\circ} & 0^{\circ} & -
\end{array}
$$

(see the sign of the trigonometry functions in the three terms of the off-diagonal elements of (7) and (8)).

Should a phase shift of $90^{\circ}$ used for the second pulse, the above phase cyclings become

$$
\begin{array}{ccc}
\beta_{0} & \beta_{1} & \text { Acq } \\
\hline 0^{\circ} & 90^{\circ} & - \\
0^{\circ} & 270^{\circ} & -
\end{array}
$$

Or alternatively

$$
\begin{array}{lcc}
\beta_{0} & \beta_{1} & \text { Acq } \\
\hline 0^{\circ} & 90^{\circ} & - \\
180^{\circ} & 90^{\circ} & +
\end{array}
$$

The change of the acquisition sign anticipates what will be demonstrated about the refocusing process (echo formation). Indeed, we can combine (11) and (13) to arrive at the four-step phase cycle, known in NMR as EXORCYCLE [17]. 


\begin{tabular}{ccc}
$\beta_{0}$ & $\beta_{1}$ & Acq \\
\hline $0^{\circ}$ & $0^{\circ}$ & + \\
$0^{\circ}$ & $180^{\circ}$ & + \\
$0^{\circ}$ & $90^{\circ}$ & - \\
$0^{\circ}$ & $270^{\circ}$ & -
\end{tabular}

Or, alternatively, by combining (12) and (14)

\begin{tabular}{lrr}
$\beta_{0}$ & $\beta_{1}$ & Acq \\
\hline $0^{\circ}$ & $0^{\circ}$ & + \\
$180^{\circ}$ & $0^{\circ}$ & - \\
$0^{\circ}$ & $90^{\circ}$ & - \\
$180^{\circ}$ & $90^{\circ}$ & +
\end{tabular}

In the same way as for $T_{1}$ measurements, we can notice that the signal is maximum for $\beta_{0}=\frac{\pi}{2}$ and $\beta_{1}=\pi$ (which would be the classical Hahn echo in NMR). Again, we would have to consider an average calculated over all the microcrystallites of the sample.

We turn now to quantities which can be refocused and thus capable of producing a spin echo. These quantities correspond to transitions for which variations lead to a slightly different resonance frequency (what we have called before $\omega^{*}$ ). Although an echo would be formed for a single $\omega^{*}$, we shall actually consider two resonances at $\omega^{*}$ and $-\omega^{*}$. This is experimentally more realistic and, for symmetry reasons, simplifies the forthcoming calculations. In that case, we obtain from equation (7) with the phase cycle (11)

$$
\sigma_{1}^{+}\left[\left(\beta_{1}\right)_{0}\right]=\left(\begin{array}{cc}
2 \sin ^{2} \frac{\beta_{0}}{2} \cos \beta_{1} e^{\frac{-\tau}{T_{1}}}+2 \sin ^{2} \beta_{1} & i \sin \beta_{0} \cos \beta_{1} e^{\frac{-\tau}{T_{2}}} \cos \omega^{*} \tau \\
-i \sin \beta_{0} \cos \beta_{1} e^{\frac{-\tau}{T_{2}}} \cos \omega^{*} \tau & -2 \sin ^{2} \frac{\beta_{0}}{2} \cos \beta_{1} e^{\frac{-\tau}{T_{1}}}+2 \sin ^{2} \beta_{1}
\end{array}\right)
$$

and from equation (8) with the phase cycle (13) 


$$
\sigma_{1}^{+}\left[\left(\beta_{1}\right)_{90}\right]=\left(\begin{array}{cc}
2 \sin ^{2} \frac{\beta_{0}}{2} \cos \beta_{1} e^{\frac{-\tau}{T_{1}}}+2 \sin ^{2} \beta_{1} & i \sin \beta_{0} e^{\frac{-\tau}{T_{2}}} \cos \omega^{*} \tau \\
-i \sin \beta_{0} e^{\frac{-\tau}{T_{2}}} \cos \omega^{*} \tau & -2 \sin ^{2} \frac{\beta_{0}}{2} \cos \beta_{1} e^{\frac{-\tau}{T_{1}}}+2 \sin ^{2} \beta_{1}
\end{array}\right)
$$

In order to get some insight into the process of echo formation, it is convenient to derive theoretical expression for the actually observed signal. It will be proportional either to $\left\langle I_{x}(t)\right\rangle$ or to $\left\langle I_{y}(t)\right\rangle$ (until now, for the sake of simplicity, we looked at $\sigma_{12}(t)$ ). In fact, $\left\langle I_{x}\right\rangle=\operatorname{Tr}\left(\sigma I_{x}\right)$ and $\left\langle I_{y}\right\rangle=\operatorname{Tr}\left(\sigma I_{y}\right)$ depend solely on $\sigma_{12}$ and $\sigma_{21}$ (see below). As the origin of the variable $t$ corresponds to the end of the $\beta_{1}$ pulse and if one takes into account the phase cycle, we obtain for $\left(\beta_{1}\right)_{0}$ and from eq. (7)

$$
\sigma_{12}(t)=\frac{i}{2}\left[\sin \beta_{0} \cos ^{2} \frac{\beta_{1}}{2} \cos \omega^{*}(\tau+t)-\sin \beta_{0} \sin ^{2} \frac{\beta_{1}}{2} \cos \omega^{*}(\tau-t)\right] e^{\frac{-(\tau+t)}{T_{2}}}
$$

and its complex conjugate for $\sigma_{21}(t)$. For $\left(\beta_{1}\right)_{90}$ the only change concerns the sign of the second term.

Altogether we obtain

$$
\begin{aligned}
& \left\langle I_{x}(t)\right\rangle=\sigma_{12}(t)+\sigma_{21}(t)=0 \\
& \left\langle I_{y}(t)\right\rangle=i\left[\sigma_{12}(t)-\sigma_{21}(t)\right]=-e^{\frac{-(\tau+t)}{T_{2}}} \sin \beta_{0}\left[\cos ^{2} \frac{\beta_{1}}{2} \cos \omega^{*}(\tau+t) \pm \sin ^{2} \frac{\beta_{1}}{2} \cos \omega^{*}(\tau-t)\right]
\end{aligned}
$$

The sign \pm in front of the second term corresponds respectively to $\left(\beta_{1}\right)_{90}$ or to $\left(\beta_{1}\right)_{0}$. In (18), we can notice that the first term corresponds to a signal produced by the first pulse while the second term is consistent with an echo formation at $t=\tau$ with different signs according to the phase $\left(0^{\circ}\right.$ or $\left.90^{\circ}\right)$ of the $\beta_{1}$ pulse. Moreover, it can be seen that if $\beta_{1}$ is equal to $\pi$ (as this is the case in NMR), the first term is actually zero. As far as NQR is concerned, $\beta_{1}$ (as well as $\beta_{0}$ ) is different from one microcrystallite to the other and an integration must 
be performed over all the possible orientations. Referring to equations (1) and (2), and defining $A$ and $B$ by

$$
\begin{aligned}
& A=\frac{1}{4 \pi} \int_{0}^{\pi} \int_{0}^{2 \pi} \sin \left[\alpha_{0} f(\theta, \varphi)\right] \sin ^{2}\left[\frac{\alpha_{1}}{2} f(\theta, \varphi)\right] f(\theta, \varphi) \sin \theta d \theta d \varphi \\
& B=\frac{1}{4 \pi} \int_{0}^{\pi} \int_{0}^{2 \pi} \sin \left[\alpha_{0} f(\theta, \varphi)\right] \cos ^{2}\left[\frac{\alpha_{1}}{2} f(\theta, \varphi)\right] f(\theta, \varphi) \sin \theta d \theta d \varphi
\end{aligned}
$$

We obtain

$$
\begin{aligned}
& \left\langle I_{y}(t)\right\rangle_{e f f}^{\left(\beta_{1}\right)_{90}}=-e^{\frac{-(\tau+t)}{T_{2}}}\left[A \cos \omega^{*}(\tau-t)+B \cos \omega^{*}(\tau+t)\right] \\
& \left\langle I_{y}(t)\right\rangle_{e f f}^{\left(\beta_{1}\right)_{0}}=e^{\frac{-(\tau+t)}{T_{2}}}\left[A \cos \omega^{*}(\tau-t)-B \cos \omega^{*}(\tau+t)\right]
\end{aligned}
$$

Again with the assumptions made above (pseudo $90^{\circ}$ pulse for $\delta_{0}$ and pseudo $180^{\circ}$ pulse for $\delta_{1}$ ), we obtain numerically (Bessel functions, frequently invoked in NQR theory [18], cannot be used for these integrals)

$$
\begin{aligned}
& \text { for } \eta=0: A=0.698, B=0.051 \\
& \text { for } \eta \neq 0: A=0.359, B=0.077
\end{aligned}
$$

As already mentioned, only the first term $\left(A \cos \omega^{*}(\tau-t)\right)$ would prevail in a spin echo NMR experiment. The signal would be maximum for $t=\tau$ regardless of the value of $\omega^{*}$, that is for all possible extra resonances. This represents of course the echo formation. Here, because of the second term $\left( \pm B \cos \omega^{*}(\tau+t)\right)$, this property no longer holds and the echo may no longer occur at $t=\tau$, as long as we employ a two-step phase cycle (see (11)-(14)). However, if we have recourse to a four-step phase cycle (see (15) or (16)), the second term of (21) and (22) vanishes and a "normal” situation is retrieved and the echo does occur at $t=\tau$. 
Echo formation in the case of a two-step phase cycle as observed in the magnitude mode

We have performed many experiments in the magnitude mode with a two-step phase cycle. The magnitude mode was chosen in order to measure accurately the echo maximum and its height, and to get rid of the oscillations which result from a difference between the carrier frequency and the resonance frequency (which can occur in the course of the experiment due to slight temperature variations). In these conditions, we observed echo shifts and some other peculiarities. It is the purpose of this section to understand these features.

Let us first consider a simple doublet made of two resonances at frequencies $+\omega^{*}$ and $-\omega^{*}$. From equations (21) and (22), we know that the signal after the second pulse can be expressed as (omitting the $T_{2}$ relaxation damping factor and with the phase cycles (11) and (13))

$$
S(t)=A \cos \omega^{*}(t-\tau) \pm B \cos \omega^{*}(t+\tau)
$$

The sign in front of the second term depends on the phase of the $\beta_{1}$ pulse : plus for $90^{\circ}$ and minus for $0^{\circ}$ (see (21) and (22)).

It can be recalled that the time origin is set at the end of the second pulse. If $S(t)$ is actually measured, an average on all possible values of $\omega^{*}$ has to be done. Provided that $(t+\tau)$ is sufficiently long, the second term will cancel, while the first term will be maximum at $t=\tau$ (echo formation, as expected). Therefore, except for short $\tau$ values (where the second term does not average to zero), nothing particular is expected if the signal is measured classically in the time domain.

However, considerable changes occur if the signal is measured in the magnitude mode. We have seen before that $\left\langle I_{x}(t)\right\rangle$ is zero so that the observed quantity will be $\sqrt{\left(\left\langle I_{y}(t)\right\rangle\right)^{2}}$. Thus, in order to detect at which time the echo is formed, we have just to consider the square of (23) $[S(t)]^{2}=\left[\left\langle I_{y}(t)\right\rangle\right]^{2}=A^{2} \cos ^{2} \omega^{*}(t-\tau)+B^{2} \cos ^{2} \omega^{*}(t+\tau) \pm 2 A B \cos \omega^{*}(t-\tau) \cos \omega^{*}(t+\tau)$ 
Let us first focus on the last term. It can be expressed as $\pm 2 A B\left(\cos 2 \omega^{*} t+\cos 2 \omega^{*} \tau\right)$. As for the second term of (23) and due to the distribution of $\omega^{*}$, these two quantities average to zero (or nearly zero) provided that the products $2 \omega^{*} t$ and $2 \omega^{*} \tau$ are sufficiently large, that is if they vary by much more than $2 \pi$. This, of course, is not true for the square of the cosine functions. We are thus left with the first two terms on the right hand-side of (24). An immediate consequence is that the observed signals arising from $\left(\beta_{1}\right)_{0}$ or $\left(\beta_{1}\right)_{90}$ will be strictly identical, if $\tau$ and $t$ are sufficiently long .

It is apparent from (23)-(24) that refocusing for the considered doublet will occur at a time ( $\left.t_{\text {echo }}\right)$ which depends on $\omega^{*}$. To find $t_{\text {echo }}$, we have just to set to zero the derivative of

$$
E^{2}(t)=A^{2} \cos ^{2} \omega^{*}(t-\tau)+B^{2} \cos ^{2} \omega^{*}(t+\tau)
$$

We arrive at

$$
\tan \left(2 \omega^{*} t_{\text {echo }}\right)=\frac{A^{2}-B^{2}}{A^{2}+B^{2}} \tan \left(2 \omega^{*} \tau\right)
$$

As already stated, when the second term does not exist (as this is the case for an NMR experiment), the echo occurs at $t_{\text {echo }}=\tau$. Here, due to the fact that, in all cases, $B^{2}<A^{2}$ and referring to (26), we can note that one has always (regardless of the values of $\omega^{*}$ )

$$
t_{\text {echo }} \leq \tau
$$

Moreover, due to the shape of the tangent function, it is easy to see that the shift between $t_{\text {echo }}$ and $\tau$ increases when $\tau$ increases. However, because we ignore the distribution function of $\omega^{*}$, we do not report in detail a series of simulations (actually performed with a Gaussian distribution) which nevertheless indicates that the reduced echo amplitude $E(t)$ (without $T_{2}$ attenuation) does not significantly depends on $\tau$. Therefore, experimentally, we should be able to measure the attenuation factor $\exp \left(-\frac{\tau+t_{\text {echo }}}{T_{2}}\right)$. 


\section{Experimental verifications}

All experiments have been performed with a home-made spectrometer described before [19]. The output of the power amplifier was limited to 100W. HMT or sodium nitrite powders were inserted into $10 \mathrm{~mm}$ o.d. NMR tube filled approximately at $5 \mathrm{~cm}$ height. The probe was placed in an insulation box and maintained at ambient temperature, so as to minimize temperature variations. For the high frequency lines (3 and $4 \mathrm{MHz}$ ), 500 transients were accumulated, whereas for the $1 \mathrm{MHz}$ line, 5000 transients were necessary. Pseudo $90^{\circ}$ or $180^{\circ}$ pulses correspond to $\alpha_{90^{\circ}}=119.5^{\circ}, \alpha_{180^{\circ}}=257^{\circ}$ for compounds with $\eta \neq 0\left(\mathrm{NaNO}_{2}\right)$ and $\alpha_{90^{\circ}}=101.5^{\circ}, \alpha_{180^{\circ}}=208^{\circ}$ for compounds with $\eta=0$ (HMT) [13]. $\alpha$ is defined in eq. 1. The corresponding pulse length $\delta$ are given, for each frequency, in tables1 and 2.

\section{1. $T_{1}$ measurements}


As indicated in the theoretical section, experiments were carried out according to the pseudo inversion-recovery method: (pseudo $180^{\circ}$ )- $\tau$ - (pseudo $90^{\circ}$ )-Acquisition. A typical recovery, involving the phase cycling indicated in the theoretical section, is shown in figure 2. We can notice that this curve does not start at a value opposite of the maximum at high $\tau$ values. This is of course due to the fact that $\alpha_{0}$ is not a true $180^{\circ}$ pulse but a "pseudo $180^{\circ}$ pulse" such that the integration over all microcrystallites is zero. Anyhow, for all cases considered (the three lines of $\mathrm{NaNO}_{2}$ and the single line of HMT; see figure 3), the results given in table 1 are of the same order of magnitude as literature data [2,20-22] when they exist (for instance, we were unable to find a $T_{1}$ value for the $1 \mathrm{MHz}$ transition of $\mathrm{NaNO}_{2}$ ).

Some comments must be done about the first part of the recovery. As shown in figure 4, without phase cycling, a very particular behavior is observed, attributable to the first two terms in the off-diagonal elements of the density matrices (7) and (8). Since these two terms, are damped by $T_{2}$ (or $T_{2}^{*}$ ) they disappear relatively quickly. However, still from figure 4, we see that perfectly clean results, even at the beginning of the recovery, are obtained with phase cycling (9) or (10). It thus appears that, with a proper phase cycling, there is strictly no problem for measuring a meaningful $T_{1}$ from recoveries which anyhow seem to be systematically monoexponential. Of course, determining $T_{1}$ from the initial slopes of recovery curves requires the asymptote value which can be obtained by performing the experiment with a very large $\tau$ value or, more simply, with a one-pulse sequence. Although there exist two $T_{1}$ relaxation times [20,23], their respective weights are such that, in most cases, only one of them contribute to the recovery curve, hence the monoexponential character of the latter [5] . On the other hand, as far as the $\omega_{x}$ transition is concerned, the measured $T_{1}$ does not seem to significantly vary with temperature, at least in the range that we have investigated (210-350 K) [24]. 


\section{2. $T_{2}$ measurements}

As in NMR, we can hope to derive a value of $T_{2}$ from the decay of echo amplitudes, the echoes being measured in the magnitude mode. However, as anticipated in the previous section, we met some difficulties with the two-step phase cycles (11) and (13). They will first be described. A typical series of echoes measured with the phase cycle (11), thus devoid of the unwanted terms involving $T_{1}$ (see (7) and (8)), is shown in figure 5. They concern $\mathrm{NaNO}_{2}$ and, in that case (non-zero asymmetry parameter), the coefficient $B$ of equations (23)-(26) is not negligible with respect to $A$, therefore the theory developed before fully applies. This figure is an indisputable illustration of the echo shift, which is seen to increase when $\tau$ increases (it is roughly $5 \%$ of $\tau$ ), another feature predicted by the theory. A further peculiarity, associated with the two-step phase cycles (11) and (13), is the initial behavior of the decay curves in the case of $\mathrm{NaNO}_{2}$. It was predicted that when the third term of (24) is not average to zero (short $\tau$ values), this extra term would be negative when the phase of the second pulse is $0^{\circ}$ and positive when it is $90^{\circ}$. This is exactly what is observed in figure 6 where the three top traces represent the decay curves of the $\mathrm{NaNO}_{2}$ line at $3.6 \mathrm{MHz}$ under the three phase cycles (11), (13) and (15). It can be noted that the four-step phase cycle (15) leads to an almost normal behavior. However, even though the decay is cleaner, we still observed in that case an echo shift. This can be due to transmitter phase imperfections or to a skew echo shape as suggested in reference [7]. This asymmetric shape (possibly due to irreversible $T_{2}$ relaxation) could as well result in an effective echo shift.

Our theory predicted also that, in the case of a zero asymmetry parameter, nothing special would happen due to the fact that the coefficient $B$ is much smaller than $A$. Again, this is perfectly verified in figure 6 (bottom) in the case of HMT. The three traces (corresponding to the three phase cycles) are seen to nearly coincide. However what is not shown in the figure is 
a constant echo shift (independent of $\tau$ ) that we shall denote $\tau_{0}$ with $t_{\text {echo }}=\tau-\tau_{0}$. This constant shift (of $0.5 \mathrm{~ms}$ ) could again be ascribed to the skew echo shape [7].

We can now establish the recipes for measuring $T_{2}$ in optimal conditions: use the four-step phase cycle, disregard nevertheless data at the beginning of the decay curve, observe the echo in the magnitude mode, measure its amplitude, determine $t_{\text {echo }}$ and plot the data as a function of $\tau+t_{\text {echo }}$. In these conditions, we were able to observe, in all cases considered here, a monotonic decay which is seen to be monoexponential, thus, leading to a definite $T_{2}$ value. This is shown in figure 7, again for the three lines of $\mathrm{NaNO}_{2}$ and for the single line of HMT. In each case, data at the beginning of the evolution have been disregarded (and not shown in this figure). The values found for $T_{2}$ are given in table 2 together with the experimental parameters. Compared to literature values, our $T_{2}$ 's are of the same order of magnitude, but shorter in the case of HMT: 1.1ms [8], 1.5ms [25] and larger for the $\omega_{x}$ transition of $\mathrm{NaNO}_{2}$ : 5ms [21], 5.3ms [22], 4.5ms [26] (data concerning the two other lines could not be found). This is not quite surprising since it is well known that NQR line-shapes and line-widths can be sample dependent [27]. Although the interpretation of relaxation times is beyond the scope of this study, we can notice the difference between $T_{2}$ and $T_{2}^{*}$ (of course, $T_{2}$ is always larger and expected than $T_{2}^{*}$ ): for $\mathrm{NaNO}_{2}$, it is much more important at the two high frequency transitions than at the low frequency transition. Surprisingly, HMT $T_{2}$ and $T_{2}^{*}$ are of the same order of magnitude although the resonance occurs at a relatively high frequency.

\section{Summary and Conclusion}

The main objective of this work was to assess the capabilities of two-pulse sequences for measuring spin 1 (as ${ }^{14} \mathrm{~N}$ ) NQR relaxation times of powder samples (by opposition to single 
crystals). Transposing NMR methods is precisely hampered by the lack of a preferential direction (contrary to the static magnetic field direction in NMR). This entails a number of particularities in NQR resulting from the necessity of integrating over the random directions of microcrystallites constituting the sample. By means of extensive theoretical developments, experimentally verified, we can propose several recipes for reliably determining the two NQR relaxation times. We first recall that true $90^{\circ}$ or $180^{\circ}$ pulses do not exist ( again because of the random orientation of microcrystallites) although we can always define pseudo $90^{\circ}$ and $180^{\circ}$ pulses which correspond to the first maximum and the first zero of nutation curves, respectively.

The well known NMR inversion-recovery experiment can be substituted by the sequence $\left(\right.$ pseudo180 $\left.{ }^{\circ}\right)-\tau-\left(\right.$ pseudo90 $\left.{ }^{\circ}\right)-($ signal acquisition $)$, where $\tau$ stands for the evolution interval. With an appropriate phase cycling, a recovery curve involving $T_{1}$ as time constant is actually obtained. One has not to worry that, contrary to the NMR experiment, the signal does not start at $-M_{0}$ but at a value greater than $-M_{0}$ ( $M_{0}$ is the signal value for $\tau$ infinite). Thus this experiment is quite trustable, as far as $T_{1}$ determination is concerned.

In NMR $T_{2}$ can be measured by the well-known Hahn spin echo sequence. In NQR, this sequence would become $\left(\right.$ pseudo $\left.90^{\circ}\right)-\tau-\left(\right.$ pseudo $\left.180^{\circ}\right)-t_{\text {echo }}($ signal acquisition $)$. Our theoretical considerations (experimentally verified) indicate that - as for inversion-recovery, a proper phase cycling must be implemented - data at the beginning of the decay may be corrupted in spite of this phase cycling and have to be disregarded until an monotonic decay is reached.

- a major difference with the NMR experiment is the time $t_{\text {echo }}$ at which the echo maximum is formed. It is always smaller than (or equal to) $\tau$, depending on the chosen phase cycle or to other phenomena (e.g. skew echo shape). In order to properly estimate $t_{\text {echo }}$, it is mandatory to 
process data in the magnitude mode (so as to avoid any effect due to a difference between the carrier and the resonance frequencies). Once $t_{\text {echo }}$ has been determined data can be fitted according to $\exp \left(-\frac{\tau+t_{\text {echo }}}{T_{2}}\right)$. Consequently, confident $T_{2}$ values can be determined however at the expense of some precautions. The next step will be to carry out a similar study about $T_{2}$ measurements derived from pulse trains ( for instance an experiment derived from the well known NMR Carr-Purcell-Meiboon-Gill sequence).

This work is part of the ANR project "Instrumentation in Magnetic Resonance" (Grant Blan06-2_139020) 


\section{References}

[1] see for instance : D.Canet, Nuclear Magnetic Resonance: Concepts and methods ;

Chichester : Wiley (1996).

[2] S. Alexander, A. Tzalmona ; Phys. Rev., A138, 845 (1965).

[3] T.N Rudakov, V.T. Mikhaltsvitch ; Chem. Phys. Lett., 363,1 (2002).

[4] T.N. Rudakov, P.A. Hayes, V.T. Mikhaltsevitch ; Phys. Lett., 330, 280 (2004).

[5] T.N. Rudakov, P.A. Hayes, V.T. Mikhaltsevitch, and W.P. Chisholm ; Appl. Magn. Reson., 25, 501 (2004).

[6] J.L. Schiano, A.J. Blauch, M.D. Ginsberg ; Z. Naturforsch., 55, 67 (2000).

[7] K.L. Sauer, B.H. Suits, A.N. Garroway, J.B. Miller ; J. Chem. Phys., 118, 5071(2003).

[8] G. Ota, H. Itozaki ; Solid State Nucl. Magn. Reson., 30, 135 (2006).

[9] S.Vega ; J. Chem. Phys., 61, 1093 (1974).

[10] C.A. Meriles ; J. Magn. Res., 149, 188 (2001).

[11] Y.K.Lee ; Conc.Magn.Res., 14, 155 (2002).

[12] B.Cordier, D.Grandclaude, A.Retournard, L.Merlat, D.Canet ; Molec. Phys., 103, 2593 (2005).

[13] D.Canet, L.Merlat, B.Cordier, D.Grandclaude, A.Retournard, M.Ferrari ; Molec. Phys., 104, 1391 (2006).

[14] M.Ferrari, N. Hiblot, A. Retournard, D. Canet ; Molec. Phys., 105, 3005 (2007).

[15] R.S. Cantor, J.S. Waugh ; J. Chem. Phys., 73, 1054 (1980).

[16] X. Youlin, Y. Chaohui ; Prog. Natural Science, 6, 284 (1996).

[17] G.Bodenhausen, R.Freeman, D.L.Turner ; J. Magn.Res., 27, 511 (1977).

[18] J.B. Miller, B.H. Suits, A.N. Garroway; J. Magn.Res., 151, 228 (2001).

[19] N. Hiblot, B. Cordier, M. Ferrari, A. Retournard, D. Grandclaude, J. Bedet, S. Leclerc,

D. Canet ; C.R. Chimie, 11, 568 (2008).

[20] G. Petersen, P.J Bray ; J. Chem. Phys., 64, 522 (1976).

[21] T.N. Rudakov, P.A. Hayes, W.P. Chisholm; Hyperfine Interactions, 59, 131 (2004) 
[22] V.T. Mikhaltsevitch; J. Magn. Reson., 117, 173 (2005)

[23] Y. Abe ; J. Phys. Soc. Japan, 23, 51 (1967).

[24] M. Ferrari, PhD thesis, Université Henri Poincaré, (2008).

[25] T.N. Rudakov, V.T. Mikhaltsevitch, J.H. Flexman; Solid State Nucl. Magn. Reson., 25, 112 (2004)

[26] V.T. Mikhaltsevitch, T.N. Rudakov, J.H. Flexman, P.A. Hayes, W.P. Chisholm; Solid State Nucl. Magn. Reson., 25, 61 (2004)

[27] M.L. Buess, S. M. Caulder; Appl. Magn. Reson., 25, 383 (2004) 


\section{Tables}

Table 1. Experimental parameters used for the measurement of $\mathrm{T}_{1}\left(\delta_{0}\right.$ and $\delta_{1}$ are the durations of the pseudo $\pi$ and pseudo $\frac{\pi}{2}$ pulses) for the three $\mathrm{NaNO}_{2}$ lines and for the single HMT line.

Experimental data (see figure 3) have been fitted according to $A\left(1-k e^{\frac{-\tau}{T_{1}}}\right)$.

\begin{tabular}{|c|c|c|c|c|}
\cline { 2 - 5 } \multicolumn{1}{c|}{} & \multicolumn{3}{c|}{$\mathrm{NaNO2}$} & $\mathrm{HMT}$ \\
\cline { 2 - 5 } \multicolumn{1}{c|}{} & $\omega_{\mathrm{x}}=4.64 \mathrm{MHz}$ & $\omega_{\mathrm{y}}=3.6 \mathrm{MHz}$ & $\omega_{\mathrm{z}}=1.04 \mathrm{MHz}$ & $\omega=3.3 \mathrm{MHz}$ \\
\hline$\delta_{0}(\mu \mathrm{s})$ & 28 & 25 & 46 & 19 \\
\hline$\delta_{1}(\mu \mathrm{s})$ & 11 & 10 & 14 & 8 \\
\hline $\mathrm{T}_{1}(\mathrm{~ms})$ & 107 & 259 & 190 & 27 \\
\hline$A$ & 95.23 & 80.29 & 14.76 & 42.09 \\
\hline$k$ & 1.44 & 1.49 & 1.83 & 1.24 \\
\hline
\end{tabular}

Table 2.

Experimental parameters used for the measurement of $\mathrm{T}_{2}\left(\delta_{0}\right.$ and $\delta_{1}$ are the durations of the pseudo $\frac{\pi}{2}$ and pseudo $\pi$ pulses) for the three $\mathrm{NaNO}_{2}$ lines and for the single HMT line. Experimental data (see figure 7) have been fitted according to $A e^{\frac{-\tau}{T_{2}}} \cdot T_{2}^{*}$ was deduced from the linewidth at half height $(\Delta v)$ by $T_{2}^{*}=\frac{1}{\pi \Delta v}$.

\begin{tabular}{|c|c|c|c|c|}
\cline { 2 - 5 } \multicolumn{1}{c|}{} & \multicolumn{3}{c|}{$\mathrm{NaNO}_{2}$} & HMT \\
\cline { 2 - 5 } \multicolumn{1}{c|}{} & $\omega_{\mathrm{x}}=4.64 \mathrm{MHz}$ & $\omega_{\mathrm{y}}=3.6 \mathrm{MHz}$ & $\omega_{\mathrm{z}}=1.04 \mathrm{MHz}$ & $\omega=3.306 \mathrm{MHz}$ \\
\hline$\delta_{0}(\mu \mathrm{s})$ & 11 & 10 & 14 & 8 \\
\hline$\delta_{1}(\mu \mathrm{s})$ & 28 & 25 & 46 & 19 \\
\hline$T_{2}(\mathrm{~ms})$ & 10 & 7 & 3 & 0.7 \\
\hline$T_{2}^{*}(\mathrm{~ms})$ & 1.74 & 2.01 & 2.38 & 0.36 \\
\hline
\end{tabular}




\section{Figure captions}

Figure 1. Schematic representation of a two-pulse sequence with the notations used throughout.

Figure 2. A typical inversion recovery experiment performed at $3.608 \mathrm{MHz}$ ( $\omega_{y}$ line of sodium nitrite) with the phase cycling (9).

Figure 3. The recovery curves for the three lines of $\mathrm{NaNO}_{2}\left(\omega_{x}\right.$ at $4.6 \mathrm{MHz}, \omega_{y}$ at $3.6 \mathrm{MHz}$ and $\omega_{z}$ at $1.04 \mathrm{MHz}$ ) and the single line of the HMT fitted according to $A\left[1-k \exp \left(-\frac{\tau}{T_{1}}\right)\right]$ (see table 1 for the experimental settings and results). The low frequency $\omega_{z}$ line entails a low signal-to-noise ratio and thus a poorer fit, albeit with adequate $\tau$ values for properly determining $T_{1}$.

Figure 4. Typical initial $\mathrm{T}_{1}$ recovery curves $\left(\mathrm{NaNO}_{2}\right.$ at $\left.4.64 \mathrm{MHz}\right)$. Filled lozenges : without phase cycling. Empty circles: phase cycle (9), $\left(\delta_{0}\right)_{0,180}-\tau-\left(\delta_{1}\right)_{0}$ Acq. Empty triangles: phase cycle (10), $\left(\delta_{0}\right)_{0}-\tau-\left(\delta_{1}\right)_{0,180} A c q(+,-)$. Here, $\delta_{0}$ is the pseudo- $180^{\circ}$ pulse and $\delta_{1}$ the pseudo$90^{\circ}$ pulse.

Figure 5. A typical series of echoes $\left(\mathrm{NaNO}_{2}\right.$ at $\left.4.64 \mathrm{MHz}\right)$ obtained according to the following sequence: phase cycle (10), $\left(\delta_{0}\right)_{0}-\tau-\left(\delta_{1}\right)_{0,180}$ Acq. Here, $\delta_{0}$ is the pseudo-90 pulse and $\delta_{1}$ the pseudo- $180^{\circ}$ pulse. Note that, at short or medium $\tau$ values, the whole echo is truncated on the left due to the insertion of a dead time between the end of the second pulse and the beginning of the acquisition. Dotted vertical lines have been drawn at abscissa $2 \tau$ just to visualize the displacement of the echo maximum. The latter occurs always at $t_{\text {echo }}\left(t_{\text {echo }}<\tau\right)$.

Figure 6. $T_{2}$ measurements with different phase cycling. Empty circles: the four-step phase cycle (15) ; filled lozenges: the two-step phase cycle (11); filled triangles: the two-step phase cycle (13). Top: $\mathrm{NaNO}_{2}$ at $3.6 \mathrm{MHz}$; note the data scattering and the particular initial 
behavior. Bottom: HMT (3.3MHz) ; note the consistency of data regardless of the phase cycling.

Figure 7. Echo amplitude decays, obtained with the four-step phase cycle (15), for the three lines of $\mathrm{NaNO}_{2}$ and the single line of the HMT fitted according to $A \exp \left(-\frac{\tau+t_{\text {echo }}}{T_{2}}\right)$ (see table 2 for the experimental setting and results) after subtraction from the data points of the constant term arising from the noise variance (unavoidable in the magnitude mode representation). Data scattering is due to the difficulty of determining accurately the echo maximum and amplitude. 


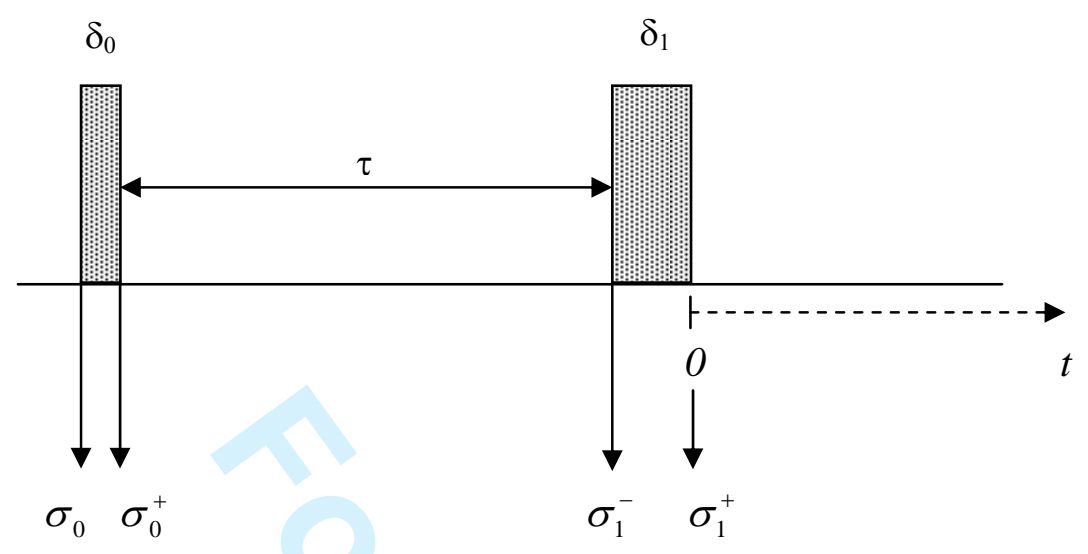

Figure 1

26
27
28
29
30
31
32
33
34
35
36
37
38
39
40
41
42
43
44
45
46
47
48
49
50
51
52
53
54
55
56
57
58
59
60




1
2
3
4
5
6
7
8
9
10
11
12
13
14
15
16
17
18
19
20
21
22
23
24
25
26
27
28
29
30
31
32
33
34
35
36
37
38
39
40
41
42
43
44
45
46
47
48
49
50
51
52
53
54
55
56
57
58
59
60

Figure 2 

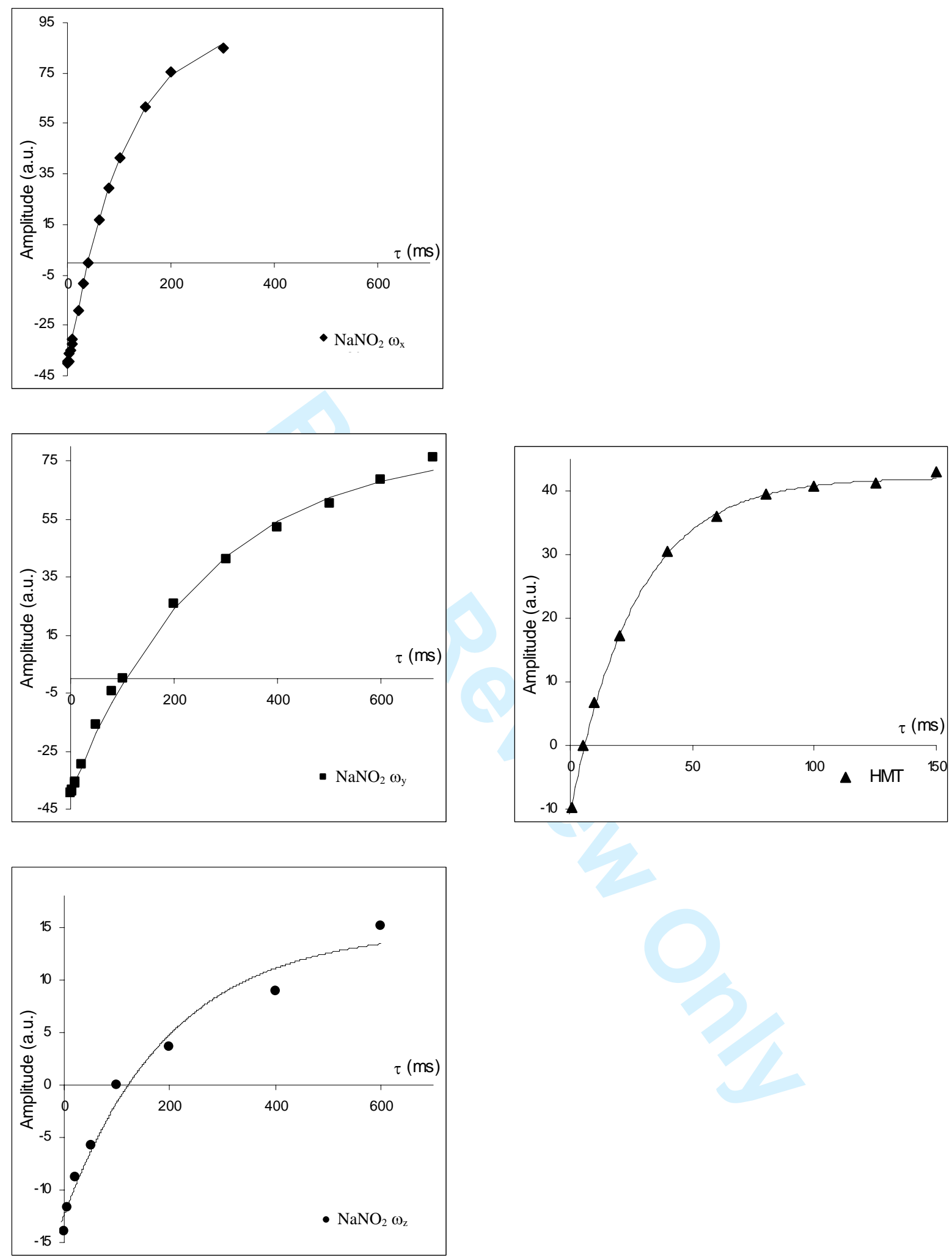

Figure 3

URL: http://mc.manuscriptcentral.com/tandf/tmph 


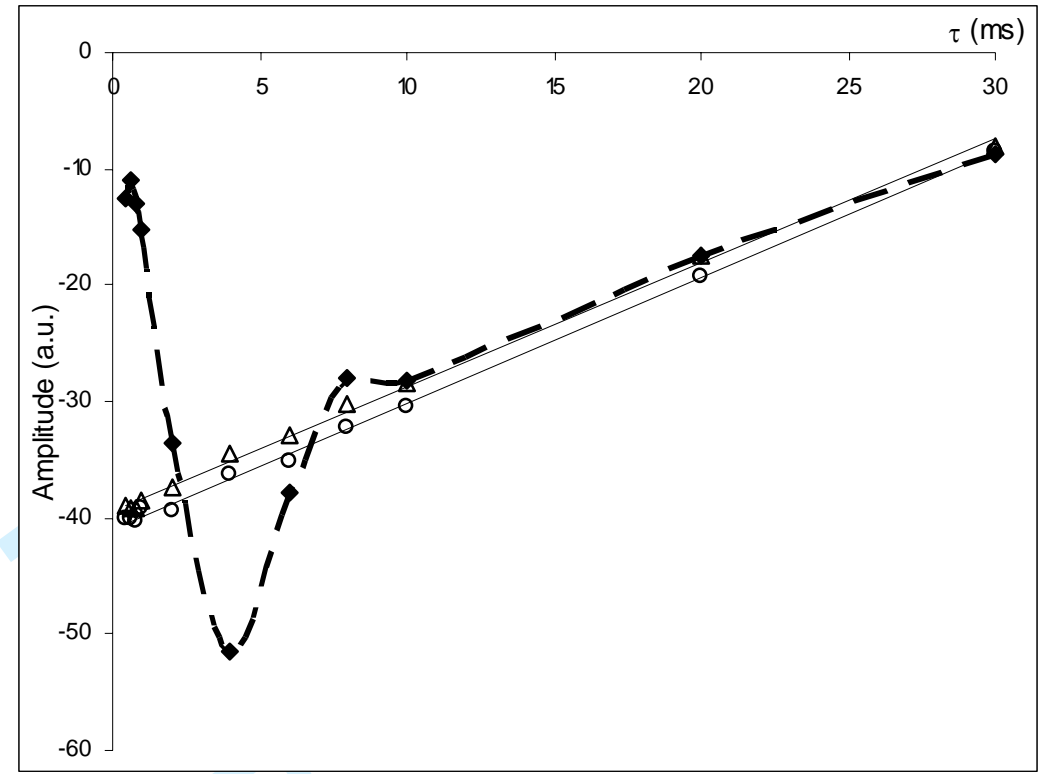

Figure 4 
Molecular Physics

Page 30 of 33

1
2
3
4
5
5
6
7
8
9
10
11
12
13
14
15
16
16
17
18
19
20
21
22
23
24
2
25
26
27
28
29
30
3
3

Figure 5

30

URL: http://mc.manuscriptcentral.com/tandf/tmph 

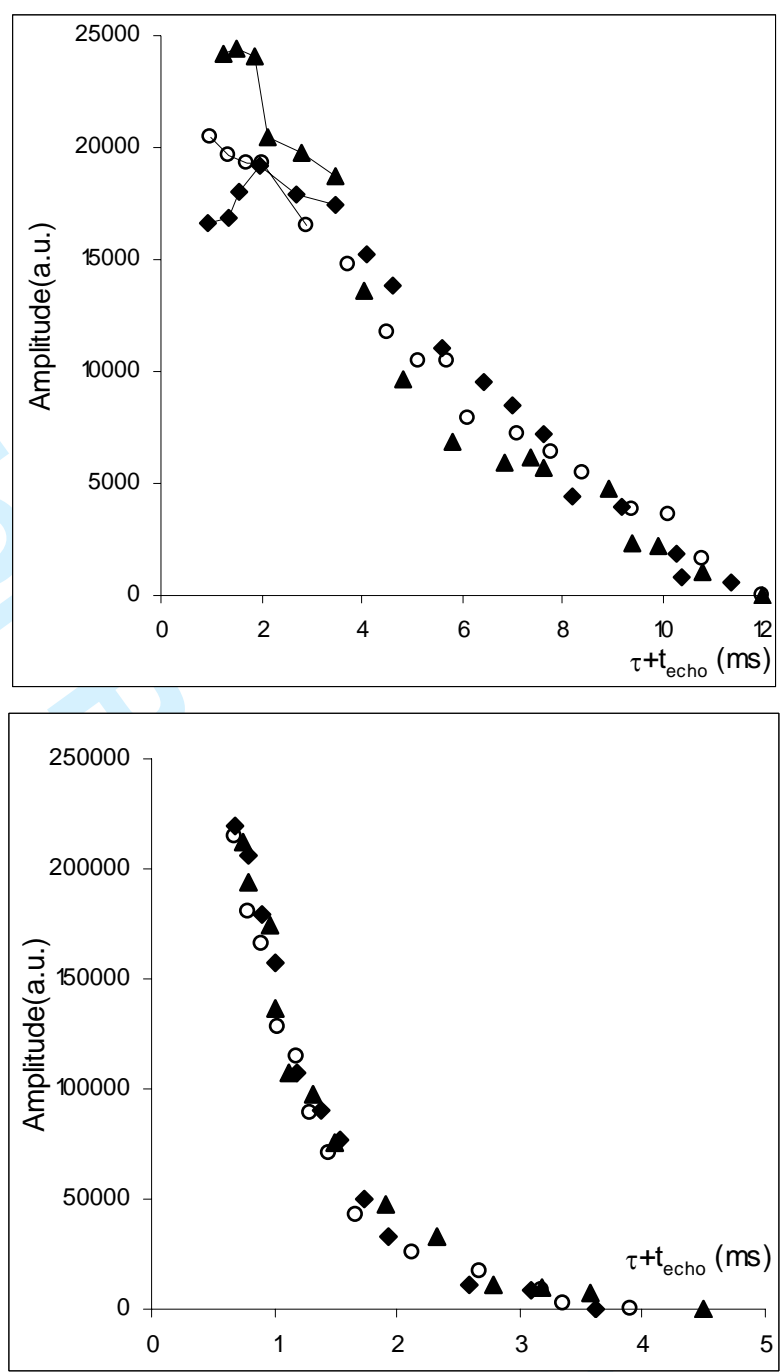

Figure 6 

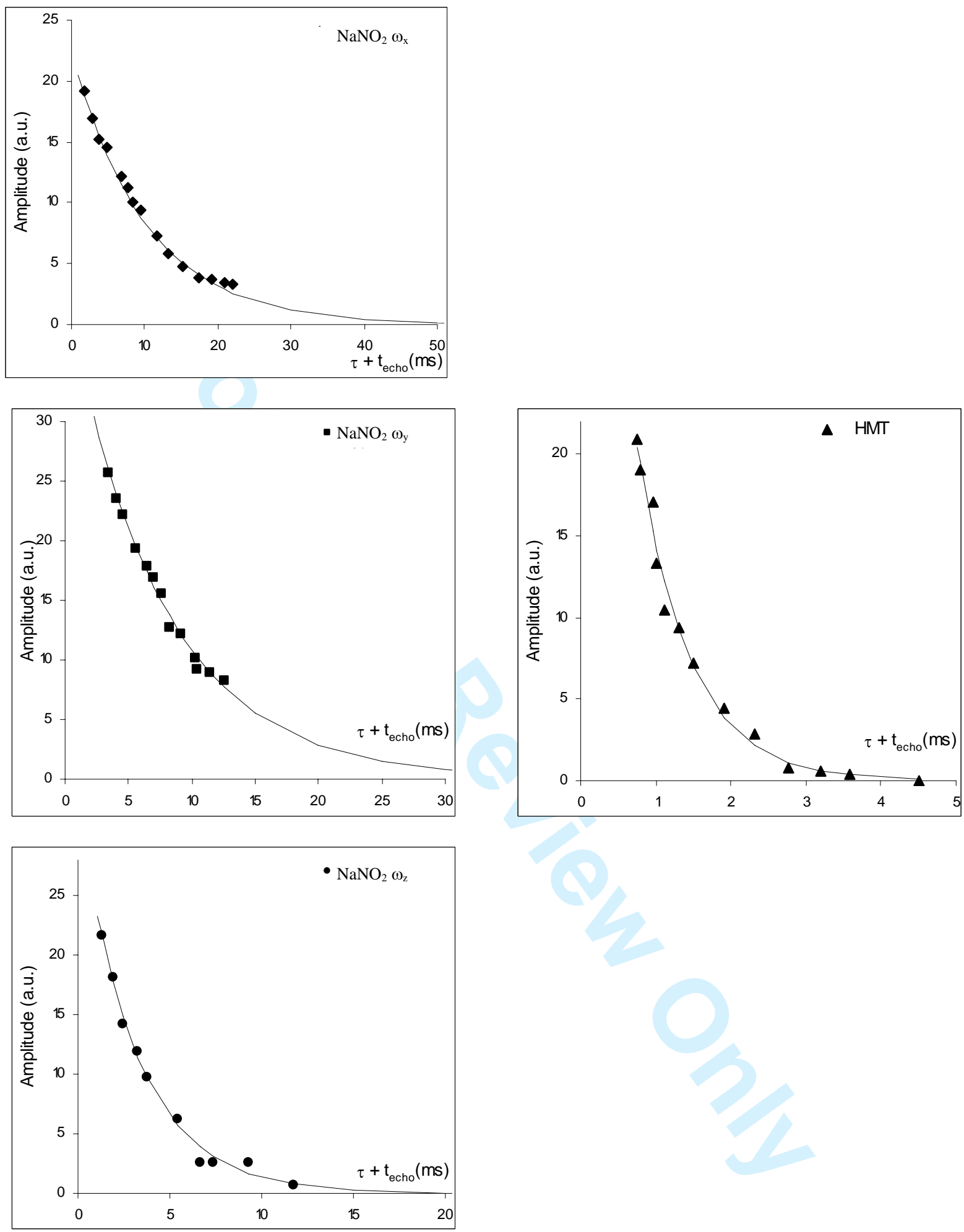

Figure 7

59 


\section{Reply to the Reviewer}

1) "homologous" has been replaced by "homolog".

2) "monotonous" has been replaced by "monotonic".

3) The clarification suggested by the Reviewer has been inserted page 4 .

4) The negligibly small temperature dependence of $T_{1}$ concerns only the $\omega_{x}$ transition of sodium nitrite while reference 2 deals only with hexamethylene tetramine (HMT).

5) Reference [2] has been corrected (page 21).

6) Reference [18] has been removed from page 16 but other references (unfortunately omitted in the previous version) have been added.

7) We totally disagree with the Reviewer and we rebut the statement "I do not find it acceptable...”. When the asymptote is known from an independent measurement, it is well known that data points at long $\tau$ are not necessary for the fit and even tend to deteriorate the result. They are in fact little sensitive to the $T_{1}$ value. Conversely, $\tau$ values around $T_{1}$ are crucial and a sentence has been added in the caption to figure 3 . We believe to know the problem relatively well and we have published several papers on the subject (see for instance two recent works: Chem. Phys. Lett. 408,237 (2005), J. Phys. Chem. A111, 10615 (2007))

8) Data of figure 6 correspond to different experiments run with different phase cycles. Empty circles correspond to the best experiment (four-step phase cycle) and exhibit actually an exponential decay. Of course, the whole set of data (including scattered data from three other experiments) could be fitted by a straight line but this would be meaningless. The results of figure 7 were obtained with the four-step phase cycle, as added in the relevant caption.

9) Literature data have been added as requested by the Reviewer. 